\title{
A Study on the Contagion Mechanism of Associated Credit Risk with Risk Information Lag
}

\author{
Kai Xu* \\ School of Management and Economics \\ University of Electronic Science and Technology of China \\ School of Business \\ Chengdu University \\ Chengdu, China \\ 363164311@qq.com
}

\author{
Zongfang Zhou \\ School of Management and Economics \\ University of Electronic Science and Technology of China \\ Chengdu, China
}

\author{
Xiaofeng Xie \\ School of Management and Economics \\ University of Electronic Science and Technology of China \\ Genenal Education Department \\ Chengdu Neusoft University \\ Chengdu, China
}

\begin{abstract}
Based on the epidemic model and complex network theory, this paper discusses the impact mechanism of risk information spreading and its lag on the associated credit risk contagion. The results of the study show that the lag of risk information do not affect the stability of risk equilibrium. Moreover, under the condition of risk stability, the density of susceptible individuals with protective awareness, the density of the infected individuals and the cumulative density of risk awareness are independent of the network structure, but are influenced by these 9 parameters such as the recovery rate of the infected individuals and the effective transmission rate of the risk information. Furthermore, the density of protected conscious susceptible individuals and the density of the infected individuals decrease with the increase of the risk awareness transmission efficiency and the effective transmission rate of risk awareness.
\end{abstract}

Keywords-Risk information lag; Associated credit risk; Contagion mechanism; Associated individual network

\section{INTRODUCTION}

In the age of mobile interconnection, information is being released and disseminated very quickly. Especially in the field of finance, there is a high degree of correlation between investment and financial products and financial institutions, the markets, investors and depositors are highly sensitive to risk information, and a little volatility can easily cause panic spread, which may lead to irreparable chain reaction. Therefore, it is very important to know the relevant risk information in advance and to prevent and control it in advance. Example in the global financial tsunami triggered by the US subprime mortgage crisis in 2008, Lehman Brothers' assets were in good shape originally, but the key factor leading to its eventual bankruptcy was the panic run sentiment. In China, the run triggered by risk information have been staged many times. In 1999, influenced by rumors of "the governor absconding with

Supported by National Natural Science Foundation of China (No.71271043), Soft Science Project of Chengdu Science and Technology Bureau (2016-RK00-00010-ZF), A Project Supported by SiChuan Landscape and Recreation Research Center (JGYQ2018004), Project of Sichuan County Economic Development Research Center (xy2018016). money", Zhengzhou Bank of Communications had triggered a run, with a daily run amounting to RMB 1 billion, seriously disrupting the bank operation order. In March of 2014, Jiangsu Sheyang Agricultural and Commercial Bank was affected by rumors that "Agricultural and Commercial Banks are going bankrupt", and thousands of people surrounded the bank's outlets to run on cash, paying 600 million yuan in cash. Above three runs were caused by information asymmetry and the lagging of official clarification. It can be seen that the individual panic run reaction caused by risk information and its lag seriously affects the economic and social harmony and stability.

At present, China's economic development has entered a new stage. There is being close and extensive, more and more complex contact among enterprises, banks, insurance companies, guarantee companies and other credit individuals, thus forming associated credit individuals. If some associated credit individuals default, resulting in the default or default probability of other related credit individuals increase, then this kind of credit risk is called associated credit risk among associated credit individuals [1]. In the end of 2009, Greece's sovereign credit was downgraded, causing the European debt crisis to erupt and then spread rapidly across the euro zone. In 2012, Zhengzhou aluminum industry loan default involves a number of banks, guarantee companies, small loan companies and hundreds of natural persons, this sudden associated credit risk made Zhengzhou guarantee industry a buzz. In the end of 2015, Yunnan coal chemical debt default, a number of banks, trusts and other financial institutions "lying gun". Above phenomenon shows that the change of associated credit risk is a linkage effect. But aren't there any clues before the risk exposure? Did many creditors not get any risk information ahead of time? Debt default continues to ferment, Northeast Special Steel Group Co., LTD. and Dalian Machine Tools Group appear one after another in 2016, many banks, trusts, 
and leasing companies are involved. It is found that the profits of the two companies had been in a downward trend of fluctuation, and both of them had delayed or even not disclosed the annual report, which was influenced by the industry cycle after 2011. But the halo of military industry, large orders and good credit ratings fooled creditors, in the macro environment of breaking the rigid payment, eventually liquidity exposure triggered bond default. Obviously, it took a long time since profits fell, until the annual report was delayed and until the first default occurred. Relevant creditors failed to grasp the risk information in time, and suffered enormous losses because of the lag of the risk information and the inertia of the good mentality. Therefore, the lag of risk information should be considered when exploring the evolutionary law of associated credit risk, identifying and preventing associated credit risk.

Associated credit individuals in this paper mainly refer to all kinds of related economies, such as cross-shareholding, credit guarantee, debt association, related transactions and so on. The network is called the associated individual network, which takes the associated individual as the node and the network is composed of the edges. In Associated individual network, if other neighbors associated with an individual infected with credit risk, the individual will estimate the probability of being infected or the loss to be suffered according to the risk information he knows and grasps, thus deciding whether to initiate risk aversion or protection measures to avoid the harm of associated credit risk to himself and even the whole network, which is called individual protection awareness. According to classical SIS model, it is assumed that there are only susceptible individuals and infected individuals in the network. If a susceptible individual receives risk information, after a period of understanding, thinking and evaluation of risk information, and so it has a sense of individual protection, which we call a susceptible individual with a sense of protection, if it doesn't has a sense of individual protection, we call it a susceptible individual without protection awareness. Risk information in this paper mainly refers to the relevant information that may have a negative impact on the individual's own credit risk, including bankruptcy liquidation, breach of contract, mutual insurance, serial guarantee, environmental protection, tax and judicial information, etc.

The transmission process of infectious diseases, Darabi etc[2]., $\mathrm{Wu}$ etc[3]. and Funk etc[4]. find the persons with protection awareness will take preventive measures such as reducing the number of persons going out, immunization and so on with the spread of this risk information in the population if they disseminate information about the emergence of infectious diseases, eventually the number of infections will be significantly reduced, and may even inhibit the outbreak of infectious diseases, so the probability of infection of susceptible individuals with protection awareness is greatly reduced. Similarly, in the contagion process of associated credit risk, that spread of risk information promote the formation of protective awareness among susceptible individuals, which in turn affects the contagion of associated credit risk. But, that susceptible individuals with protection awareness isn't considered on study of associated credit risk in the past. So, it is more realistic for risk information dissemination and susceptible individual protection awareness are included in the influencing factors of associated credit risk contagion. Will there be a lag for the spread of risk information to promote the formation of individual awareness of protection? Could we suppress the contagion of related credit risk by positively disseminating relevant risk information and regulating individual awareness? These problems are important issues in the field of the study on associated credit risk.

The contagion of credit risk among enterprises has attracted wide attention from scholars at home and abroad. Basole and Bellamy[5] studied the effects of global supply network structure on risk diffusion and supply network health and found that small world supply network topology is always superior to scale-free supply network. Jorion and Zhang[6] found that bankruptcy information can lead to abnormal stock returns and increase CDS spreads, and counterparty default is a potential channel of credit risk contagion. Boissay[7] found the risk propagates through the associated network when the asset association is formed among enterprises through commercial credit. Barro and Basso[8] constructed affiliated enterprise network and studied credit risk transmission in bank loan portfolio. It was found that business relationship among enterprises and counterparty risk would affect enterprise value. Similarly, the relationship between enterprises and banks would also lead to credit risk contagion. Delli and Gatti etc. [910], Li and Sui[11] studied the internal and external economic and financial networks composed of downstream enterprises, upstream enterprises and banks, and found that the bankruptcy risk information of enterprises and banks would spread along the network.

The spread of risk information would cause fluctuations in the value of related assets of enterprises. Ferreira and Gama[12] found that reports of the downgrade of sovereign debt had a significant negative impact on stock market returns in other countries. Jorion and Zhang[13] studied the impact of the dissemination of debt rating downgrades' information on the stock returns of portfolio stocks, and found the information asymmetry can lead to the contagion of credit risk among economic individuals. Chakrabarty and Zhang[14] found that information asymmetry accelerated the spread of credit risk among firms through studying the impact of Lehman Brothers' bankruptcy on other firms. Huang and Cheng[15] empirically analyzed the positive correlation between risk information and credit risk contagion.

The above studies show there is correlation between the spread of risk information and the spread of credit risk, but there is seldom research on the mechanism of the impact of risk information on the spread of associated credit risk. Based on the classical SIS model, this paper constructs an interaction model between the transmission of risk information and the contagion of associated credit risk in the network with credit risk and infected individuals, and use the mean field dynamics theory to study the effect of information lag on the network stability of associated individuals, and explore the influence of behavior awareness and risk information transmission efficiency on the network stability of associated individuals. Furthermore, this paper preliminarily discusses how to use risk information to control the contagion of associated credit risk. 


\section{DESIGN OF StUdy}

\section{A. Basic Hypothesis}

The evolution of associated credit risk in the network of associated individuals shows the general characteristics of infectious diseases, such as the existence of infectious sources, latency, infectivity and immunity. It is similar to infectious diseases in infectious environment, infectious process, infectious objects and infectious direction. Infectious disease models can depict the interactions between microscopic individuals based on different associations. With the rapid development of complex networks, infectious disease models have been widely used in the study of risk transmission among enterprises, diffusion of knowledge and innovation models, and spread of rumors and public opinion[16-20]. The existing research results have laid the foundation for this paper to use the infectious disease model to study the associated credit risk. For convenience of discussion, the following basic assumptions are proposed in combination with the basic characteristics of contagion of associated credit risk.

Hypothesis 1: Considering whether the susceptible individuals are protective or not, the associated individuals are divided into three categories in the network of $\mathrm{N}$ associated individuals.

Susceptible individuals without protective awareness $S^{u}$ : If the risk information is not delivered or unknown, and the susceptible individual thinks that the risk is very small and there is no need to take preventive measures, the susceptible individual is called unprotected susceptible individual. In the network of associated individuals, these susceptible individuals with low credit risk, which are susceptible to be influenced by the individuals with high credit risk associated with them, so it is great potential for these susceptible individuals to become individuals with high credit risk.

Susceptible individuals with protective awareness $S^{p}$ : Risk information has been served or known. If the susceptible individual considers that the risk is very high and it is necessary to take preventive measures after the cognition, understanding and evaluation of the information, the susceptible individual is said to be a susceptible individual with protective awareness. In associated individual network, because these individuals are aware of the risk of contagion and its harmfulness, they will take certain protective measures spontaneously. Therefore, although they are affected by the infected individuals with high credit risk associated with them, the probability of being infected is lower than that of the susceptible individuals without protection awareness.

Infected individuals $I$ : Infected individuals refer to those individuals who have high credit risk and can negatively affect the credit risk of individuals associated with them through different links. Both the structure and quantity of these individuals in the associated individual network can significantly influence the contagion effect of the associated credit risk in the associated individual network. The proportion of these individuals in the associated individual network not only represents the contagion intensity of the associated credit risk, but also shows the evolution process of the associated credit risk in the associated individual network.

Hypothesis 2: Susceptible individuals are infected by directly contact with infected individuals. A represents constant birth rate of individuals in associated networks, and these new-born individuals are all susceptible individuals without protection awareness. $B$ represents constant natural mortality rate of individuals, and is also the same mortality rate of above three kinds of credit individuals. $\alpha$ represents contact rate between susceptible individuals with protective awareness and infected individuals. $\beta$ represents contact rate between susceptible individuals without protective awareness and infected individuals. Obviously, $\alpha<\beta$. $\lambda$ represents transmission efficiency of risk awareness in susceptible individuals without protection awareness. $\gamma$ represents the transfer rate of susceptible individuals with protection awareness to susceptible individuals without protection awareness. Above parameters are all positive.

Infected individuals are restored to susceptible individuals through rescue (loan or guarantee and so on). According to whether there is protection awareness at this time, infected individuals may be restored to the susceptible individuals without protection awareness or the susceptible individuals with protection awareness. So some assumptions are following.

Hypothesis 3: The recovery rate of infected individuals recovered to susceptible individuals is $\delta$, in which the probability $q$ indicates that it is restored to a susceptible individual with protective awareness, the probability $p$ indicates that it is restored to a susceptible individual without protective awareness. Obviously, $p+q=1$.

The dissemination of risk information is obviously influenced by individual subjectivity. Especially when facing the mass information of various media and networks, individuals will have their own understanding and thinking process when they get the risk information. Moreover, a lot of risk information itself has a certain lag (such as financial crisis information, etc.) and due to the concealment of some individuals (such as profit manipulation, etc.). Although there are various channels to obtain risk information, it is still very difficult for other credit individuals to understand risk information timely and accurately. Therefore, it is necessary to consider the lag of risk information in the model. Without exception, there are following assumptions.

Hypothesis 4: Risk information spreads through the network, and susceptible individuals without protective awareness do not immediately turn into susceptible individuals with protective awareness. So introducing lag time $\tau(\tau \geq 0)$.

Risk information spreads and diffuses in the associated individual network, and forms the accumulation of individual awareness activities in the network. So some assumptions are following.

Hypothesis 5: In this paper, $M(t)$ is the cumulative density of conscious activities formed by the dissemination of risk information in the networks in the time $t$. The more 
infected individuals are, the more $M(t)$ is. $\eta$ represents effective spread rate of risk information (i.e. the risk information received by susceptible individuals is the proportion of information sources). With the time passing by, the cumulative density $M(t)$ is invalid in terms of dissipation rate $\eta_{0}$ (i.e. the proportion of risk information not received by susceptible individuals to the source of information).

\section{B. Model Establishment}

Consider that in a network consisting of $N$ associated individuals, the associated individuals are nodes in the network, and the association relations among individuals are edges in the network. The degree $k_{i}$ of associated individual $i$ is the number of individuals associated with the individual. The average degree of all individuals in the associated individual network is called the network average degree $\langle k\rangle . p(k)$ represents the probability of a randomly selected individual is exactly $k$. So, $\langle k\rangle=\sum_{i=1}^{n} k_{i} p\left(k_{i}\right)$. $S_{k}^{u}(t), S_{k}^{p}(t)$ and $I_{k}(t)$, represent the density of this three kinds of individuals with a degree of $k$ at the moment ${ }^{t}$ respectively. According to above assumptions, reference to classical SIS model, considering whether there is risk protection awareness. The dynamical system equation for contagion of associated credit risk is following,

$$
\left\{\begin{array}{l}
\frac{d S_{k}^{u}(t)}{d t}=A-\beta S_{k}^{u}(t) I_{k}(t)+\gamma S_{k}^{p}(t)-\lambda S_{k}^{u}(t) M(t-\tau)-B S_{k}^{u}(t)+p \delta I_{k}(t) \\
\frac{d S_{k}^{p}(t)}{d t}=\lambda S_{k}^{u}(t) M(t-\tau)-(\gamma+B) S_{k}^{p}(t)-\alpha S_{k}^{p}(t) I_{k}(t)+q \delta I_{k}(t) \\
\frac{d I_{k}(t)}{d t}=\beta S_{k}^{u}(t) I_{k}(t)+\alpha S_{k}^{p}(t) I_{k}(t)-(\delta+B) I_{k}(t) \\
\frac{d M(t)}{d t}=\eta I_{k}(t-\tau)-\eta_{0} M(t)
\end{array}\right.
$$

Inside, $\quad S_{k}^{u}(0)>0, \quad S_{k}^{p}(0) \geq 0, \quad I_{k}(0) \geq 0$. Because,

$$
S_{k}^{u}(t)+S_{k}^{p}(t)+I_{k}(t)=1 \text {, Substitute above equation, (1) is }
$$
translated into the following,

$$
\left\{\begin{array}{l}
\frac{d S_{k}^{p}(t)}{d t}=\lambda\left[1-S_{k}^{p}(t)-I_{k}(t)\right] M(t-\tau)-(\gamma+B) S_{k}^{p}(t)-\alpha S_{k}^{p}(t) I_{k}(t)+q \delta I_{k}(t) \\
\frac{d I_{k}(t)}{d t}=\beta\left[1-S_{k}^{p}(t)-I_{k}(t)\right] I_{k}(t)+\alpha S_{k}^{p}(t) I_{k}(t)-(\delta+B) I_{k}(t) \\
\frac{d M(t)}{d t}=\eta I_{k}(t-\tau)-\eta_{0} M(t)
\end{array}\right.
$$

Then, study the system (2) in the feasible domain $\Omega$.

$$
\Omega=\left\{\left[S_{k}^{p}(t), I_{k}(t), M(t)\right] \in R_{+}^{3}: 0 \leq S_{k}^{p}(t), I_{k}(t) \leq 1,0 \leq M(t)<\eta / \eta_{0}\right\}
$$

\section{Analysis of Risk Stability}

Definition 1: Risk stability and risk stable point: In the associated individual network, risk stability means that the contagion of associated credit risk tends to be stable after a certain period of contagion evolution. There are still infected individuals in the associated individual network at the moment, but the density of susceptible individual with protective awareness (i.e. proportion), the density of infected individual (i.e. proportion) and the cumulative density of risk information in the network are finally converged to $E^{*}\left(S^{p^{*}}, I^{*}, M^{*}\right), E^{*}$ is the risk stable point.

In this paper, we consider the existence of risk information and risk contagion in the network, and mainly study the contagion of associated credit risk without considering the state of risk-free stability. Therefore, there are the following propositions.

Proposition 1: In the associated individual network, when the contagion associated credit risk tends to be stable, the density of susceptible individual with protective awareness, the density of infected individual and the cumulative density of risk information in the network are converged to the risk stable point $E^{*}\left(S^{p^{*}}, I^{*}, M^{*}\right)$.

Prove: When considering stability, $\frac{d S_{k}^{p}(t)}{d t}=0, \frac{d I_{k}(t)}{d t}=0, \frac{d M(t)}{d t}=0$, so from (2), we can obtain,

$$
\left\{\begin{array}{l}
\lambda\left[1-S^{p^{*}}-I^{*}\right] M^{*}-(\gamma+B) S^{p^{*}}-\alpha S^{p^{*}} I^{*}+q \delta I^{*}=0 \\
\beta\left[1-S^{p^{*}}-I^{*}\right] I^{*}+\alpha S^{p^{*}} I^{*}-(\delta+B) I^{*}=0 \\
\eta I^{*}-\eta_{0} M^{*}=0
\end{array}\right.
$$

Obtained from the third equation of (3),

$$
M^{*}=\frac{\eta}{\eta_{0}} I^{*}
$$

Obtained from the second equation of (3),

$$
S^{p^{*}}=\frac{\beta\left(1-I^{*}\right)-(\delta+B)}{\beta-\alpha}
$$

Substituting (4) and (5) the first equation of (3), obtained the following equation about $I^{*}$,

$$
P_{1}\left(I^{*}\right)^{2}+P_{2} I^{*}+P_{3}=0
$$


Inside above (6), there is,

$$
\left\{\begin{array}{l}
P_{1}=\alpha\left(\lambda \eta+\eta_{0} \beta\right) \\
P_{2}=(B+\delta-\beta)\left(\lambda \eta+\alpha \eta_{0}\right)+q \delta \eta_{0}(\beta-\alpha)+\eta_{0} \beta(\beta+B) \\
P_{3}=\eta_{0}(\gamma+B)(B+\delta-\beta)
\end{array}\right.
$$

From (6), (7), Definition $R_{0}=\frac{\beta}{B+\delta}$, When $R_{0}>1$, $P_{1}>0, P_{2}<0$, there is,

$$
I^{*}=\frac{-P_{2}+\sqrt{P_{2}^{2}-4 P_{1} P_{3}}}{2 P_{1}}>0
$$

$$
\text { From (4), (5), (8), there is being } E^{*}\left(S^{p^{*}}, I^{*}, M^{*}\right) \text {. Q.E.D. }
$$

\section{Comparative Analysis of Risk Information Lag}

As can be clearly seen from Section 2.3, when the associated credit risk tends to be risk-stable, risk stable point $E^{*}\left(S^{p^{*}}, I^{*}, M^{*}\right)$ of risk information will not affect the stability of risk stable point. Obviously, in the state of risk stable, the density of susceptible individuals with protective awareness, the density of infected individuals, and the cumulative density of risk awareness are independent of the network structure, but influenced by the following 9 kinds of parameters: the constant natural mortality rate of individuals $B$, the contact rate between susceptible individuals with protective awareness and infected individuals $\alpha$, the contact rate between susceptible individuals without protective awareness and infected individuals $\beta$, the transmission efficiency of risk awareness in susceptible individuals without protection awareness $\lambda$, the transfer rate of susceptible individuals with protection awareness to susceptible individuals without protection awareness $\gamma$, the recovery rate of infected individuals recovered to susceptible individuals $\delta$, the probability of being a susceptible individual with protection consciousness $q$, the effective spread rate of risk information $\eta$, the dissipation rate of risk information $\eta_{0}$.

\section{ANALYSIS OF INFLUENCE OF PARAMETERS ON RISK STABLE POINT}

In order to study the influence of the transmission efficiency of risk awareness $\lambda$ and the effective spread rate of risk information $\eta$ on the stability of risk stable point, the method of differential dynamics is adopted, brief exploration of the effects of changes in $\lambda$ and $\eta$ on the density of susceptible and infected individuals with protective awareness.

\section{A. Influence of Parameters on Infected Individuals}

Obtained from (8), $\frac{d I^{*}}{d \lambda}<0$, indicates that the density of infected individuals decreases with the increase of the transmission efficiency of risk information at the stable point. Therefore, in order to reduce the density of infected individuals in associated individual networks, we should increase the reporting and dissemination of relevant risk information through television, broadcasting, mobile terminals, self-Media and so on, so as to improve the efficiency of risk information dissemination, and then control the outbreak of associated credit risk.

As above, obtained from (8), $\frac{d I^{*}}{d \eta}<0$, indicates that the density of infected individuals decreases with the increase of the effective spread rate of risk information at the stable point. Therefore, in order to reduce the density of infected individuals in associated individual networks, we should also increase the reporting and dissemination of relevant risk information through self-Media etc., so as to improve the efficiency of risk information dissemination, and then control the outbreak of associated credit risk.

\section{B. Influence of Parameters on Susceptible Individuals With Protective Awareness}

Obtained from (5) and (8), $\frac{d S^{p^{*}}}{d \lambda}<0$, indicates that the density of susceptible individuals with protective awareness decreases with the increase of the transmission efficiency of risk information at the stable point. So, in order to reduce the density of susceptible individuals with protective awareness in associated individual networks, we should increase the reporting and dissemination of relevant risk information through television, broadcasting, mobile terminals, self-Media and so on, so as to improve the efficiency of risk information dissemination, and then control the outbreak of associated credit risk.

As above, obtained from (5) and (8), $\frac{d S^{p^{*}}}{d \eta}<0$, indicates that the density of susceptible individuals with protective awareness decreases with the increase of the effective spread rate of risk information at the stable point. Therefore, in order to reduce the density of susceptible individuals with protective awareness in associated individual networks, we should also increase the reporting and dissemination of relevant risk information through self-Media etc., so as to improve the efficiency of risk information dissemination, and then control the outbreak of associated credit risk.

The above analysis shows that the density of susceptible individuals with protective awareness and the density of infected individuals have the same trends at the risk stable point, which decrease with the increase of the transmission efficiency of risk information and the effective spread rate of risk information. It shows that the dissemination of risk information plays a very important role in the prevention and control of associated credit risk.

\section{CONCLUSION}

In modern financial markets, the contagious evolution of associated credit risks is influenced by many factors such as the spread of risk information and its lag. Based on the previous studies of experts and scholars, the dissemination of risk information and its lagging factors are coupled into the study of associated credit risk by using infectious disease model and 
complex network theory. Based on the classical SIS model, this paper has constructed the contagion model of associated credit risk, discussed the convergence of risk stability, the lag of risk information and the influence of parameters on the stability, which has important theoretical and practical significance to reveal the contagion mechanism of associated credit risk in the network of associated individuals. The results show that the lag of risk information do not affect the stability of risk stable point Moreover, under the condition of risk stability, the density of susceptible individuals with protective awareness, the density of the infected individuals and the cumulative density of risk awareness are independent of the network structure, but are influenced by these 9 parameters such as the recovery rate of the infected individuals and the effective transmission rate of the risk information. Furthermore, the density of susceptible individuals with protective awareness and the density of the infected individuals decrease with the increase of the risk information transmission efficiency and the effective transmission rate of risk information.

The above study shows that the dissemination of risk information plays a very important role in the prevention and control of associated credit risk. Therefore, all kinds of economies in the network of associated individuals can manage the associated credit risk in the following ways: (1) Once some individuals in the associated individual network are infected with credit risk, they should actively disclose risk information, improve the dissemination of risk information, enhance the formation of protection awareness of associated individuals, reduce the density of infected individuals, and thus reduce the harm of associated credit risk. (2) Individuals in associated networks should actively collect credit risk information of other individuals who are related to them, and cut off the relationship with those individuals in the credit risk information concentration in time, so as to improve the transmission efficiency of risk information and reduce the harm of associated credit risk to associated individual networks. (3) Governments and financial institutions should formulate relevant management measures. When large-scale associated individual defaults occur, public opinion supervision should be strengthened, the dissemination and transparency of risk information should be enhanced, and the harm of associated credit risk outbreak caused by the lag of risk information should be reduced. Therefore, the conclusion of this paper has important theoretical value and practical significance for the practice of management on associated credit risk. However, the actual data of risk information in this paper is hard to collect, so we only do "throw jade" here. The collection and collation of actual data and further empirical studies are under way and will be published in the forthcoming articles.

\section{REFERENCES}

[1] Y.K. Li and Z.F. Zhou, "Contagion delayed effects of associated credit risk based on scale-free network," Journal of System Engineering, vol. 30, pp. 575-583, 2015.

[2] S.F. Darabi, F.N. Chowdhury, and C.M. Scoglio, "On the existence of a threshold for preventive behavioral responses to suppress epidemic spreading," Sci Rep, vol. 2, pp. 632, 2012.

[3] Q.C. Wu, X.C. Fu, M Small, and X.J. Xu, "The impact of awareness on epidemic spreading in networks," Chaos, vol. 22, pp. e8032-R, 2012.

[4] S. Funk, E. Gilad, and V.A.A. Jansen, "Endemic disease, awareness, and local behavioural response," Journal of Theoretical Biology, vol. 264, pp. 501-509, 2010.

[5] R.C. Basole and M.A. Bellamy, Global supply network health: Analysis and visualization, IOS Press, 2012.

[6] P. Jorion and G.Y. Zhang, "Credit Contagion and Counterparty Risk," Journal of Finance, vol. 64, pp. 2053-2087, 2009.

[7] F. Boissay, Credit chains and the propagation of financial distress. Working Paper, 2006.

[8] D. Barro and A. Basso, "Credit contagion in a network of firms with spatial interaction," European Journal of Operational Research, vol. 05, pp. 459-468, 2010.

[9] D.D. Delli, M. Gallegati, B.C. Greenwald, A. Russo, and J.E. Stiglitz, "Business fluctuations and bankruptcy avalanches in an evolving network economy,” J. Econ Interact Coord, vol. 04, pp. 195-212, 2009.

[10] D.D. Delli, M. Gallegati, B.C. Greenwald, A. Russo, and J.E. Stiglitz, "The financial accelerator in an evolving credit network," J. Econ Dyn Control, vol. 34, pp. 1627-1650, 2010.

[11] S.W. Li and W. Sui, "Contagion risk in endogenous financial networks," Chaos, Solitons and Fractals, vol. 91, pp. 591-597, 2016.

[12] M. Ferreira and P. Gama, "Does sovereign debt ratings news spill over to international stock markets," Journal of Banking and Finance, vol. 31, pp. 3162-3182, 2007.

[13] P. Jorion and G.Y. Zhang, "Information Transfer Effects of Bond Rating Downgrades," Financial Review, vol. 45, pp. 683-706, 2010.

[14] B. Chakrabarty and G.Y. Zhang, "Credit Contagion Channels: Market Microstructure Evidence from Lehman Brothers'Bankruptcy,” Financial Management, vol. 41, pp. 320-343, 2012.

[15] A.Y. Huang and C.M. Cheng, "Information risk and credit contagion," Finance Research Letters, vol. 10, pp. 116-123, 2013.

[16] X.H. Hu, L.Z. Chen, and K. Lv, "Analysys and Simulation of the knowledge Diffusion Mechanism within Industrial Clusters Based on Epidemic Mode," Operation Research and Management Science, vol.24, pp.248-257, 2015

[17] Y.Y. Wang and W. Xie, "A study on the diffusion mechanism of business models based on the Epidemic Mode," Science Rearch Management, vol.36, pp.10-18, 2015.

[18] Y.C. Zhang, Y. Liu, H.F. Zhang, H. Cheng, and F. Xiong, "The research of information dissemination model on online social network," Acta Phys.Sin. vol.60, pp.60-66, 2011.

[19] Y.Y. Ma, X.T. Zhuang, and L.X. Li, "Simulation and SIR model of crisis propagation in stock market," Journal of Management Science in China, vol.16, pp.80-94, 2013.

[20] K. Yang and Z.Y. Zhang, "Research on risk propagation mechanism of supply chain network based on complex network theory," Journal of Systems Science and Mathematical Sciences, vol.33, pp.1225-1232, 2013 . 